\title{
GENERATORS FOR THE BORDISM ALGEBRA OF IMMERSIONS
}

\author{
M. A. AGUILAR
}

\begin{abstract}
Let us denote by $I(n, k)$ the group of bordism classes of immersions of closed smooth $n$-manifolds in closed smooth $(n+k)$-manifolds $(k>$ $0)$. We can make $I(*, k)$ into a graded algebra over the unoriented bordism ring. This algebra is polynomial. In this paper we give two sets of immersions which are polynomial generators.
\end{abstract}

\section{Statement of MAin Result}

We consider immersions of closed smooth $n$-manifolds in closed smooth $(n+k)$-manifolds, $k>0, n \geq 0$. Given two immersions $f: M \rightarrow N$ and $f^{\prime}: M^{\prime} \rightarrow N^{\prime}$, we say that they are bordant if there exists an immersion $F: V \rightarrow$ $W$ such that (i) $V$ is a compact smooth $(n+1)$-manifold whose boundary $\partial V$ is diffeomorphic to the disjoint union $M \amalg M^{\prime}$, (ii) $W$ is a compact smooth $(n+k+1)$-manifold whose boundary is diffeomorphic to $N \amalg N^{\prime}$, (iii) $F \mid M=f$ and $F \mid M^{\prime}=f^{\prime}$.

We denote by $I(n, k)$ the set of equivalence classes under this relation and by $[f: M \rightarrow N]$ the equivalence class of an immersion. Letting $\mathscr{N}_{*}$ denote the unoriented cobordism ring, $I(*, k)$ is an $\mathscr{N}_{*}$-algebra with the following operations:

$$
\begin{aligned}
& {[f: M \rightarrow N]+\left[f^{\prime}: M^{\prime} \rightarrow N^{\prime}\right]=\left[f \amalg f^{\prime}: M \amalg M^{\prime} \rightarrow N \amalg N^{\prime}\right],} \\
& {[f: M \rightarrow N] \cdot\left[f^{\prime}: M^{\prime} \rightarrow N^{\prime}\right]} \\
& \quad=\left[f \times \operatorname{id} \amalg \operatorname{id} \times f^{\prime}: M \times N^{\prime} \amalg N \times M^{\prime} \rightarrow N \times N^{\prime}\right],
\end{aligned}
$$

(c) $\left[M^{\prime}\right] \cdot[f: M \rightarrow N]=\left[\operatorname{id} \times f: M^{\prime} \times M \rightarrow M^{\prime} \times N\right], \quad$ where $\left[M^{\prime}\right] \in \mathscr{N}_{*}$.

Consider the antipodal action of the symmetric group $\Sigma_{2}$ on the sphere $S^{r}$. If $X$ is any space we have a free action of $\Sigma_{2}$ on the product $S^{r} \times X \times X$ given by $\left(a, x_{1}, x_{2}\right) \mapsto\left(-a, x_{2}, x_{1}\right)$. We denote by $S^{r} \times_{\Sigma_{2}} X \times X$ the quotient space.

Received by the editors May 30, 1986.

1980 Mathematics Subject Classification (1985 Revision). Primary 57R90, 55S12, 55N22; Secondary 55P47, 55R 12.

Key words and phrases. Bordism of immersions, bordism of embeddings, infinite loop space, Dyer-Lashof operations, transfer. 
In this paper we are going to study operations $\widehat{Q}_{r}: I(n, k) \rightarrow I(2 n+k+r, k)$, $k>0, r \geq 0$, given by

$$
\widehat{Q}_{r}[f: M \rightarrow N]=\left[\hat{f}: S^{r} \times N \times M \rightarrow S^{r} \times_{\Sigma_{2}} N \times N\right]
$$

where $\hat{f}(a, y, x)=[a, y, f(x)]$. With these operations we shall construct generators for the algebra $I(*, k)$, for each $k>0$. Let $\xi=(E, p, M)$ be a smooth vector bundle. We denote by $D(\xi)$ the disc bundle and by $\widetilde{D}(\xi)$ the double of $D(\xi)$. Now consider the embedding $M \rightarrow \widetilde{D}(\xi)$ of $M$ in $\widetilde{D}(\xi)$ as the zero section.

Our main result is the following

Theorem. The algebra $I(*, k), k>0$, is a polynomial algebra over $\mathcal{N}_{*}$ on $\left\{\widehat{Q}_{i_{1}} \widehat{Q}_{i_{2}} \cdots \widehat{Q}_{i_{d}}\left[P^{\alpha_{1}} \times P^{\alpha_{2}} \times \cdots \times P^{\alpha_{k}} \rightarrow \widetilde{D}\left(\gamma_{\alpha_{1}} \times \gamma_{\alpha_{2}} \times \cdots \times \gamma_{\alpha_{k}}\right)\right]: 0<i_{1} \leq i_{2} \leq\right.$ $\left.\cdots \leq i_{d}, d \geq 0 ; 0 \leq \alpha_{1} \leq \alpha_{2} \leq \cdots \leq \alpha_{k}\right\}$, where $\gamma_{n}$ is the canonical line bundle over the real projective space $P^{n}$.

We also give a similar set of generators formed from Milnor manifolds. In $\S 2$ we define generators for the bordism of embeddings which will be used to construct the generators for $I(*, k)$. We begin $\S 3$ with a theorem [13] that gives an isomorphism between $I(*, k)$ and the bordism of an infinite loop space. Then, following $[2,12,16]$, we define Dyer-Lashof operations in homology and in bordism. In $\S 4$ we define the operations $\widehat{Q}_{r}$ and give the proof of the main theorem.

I would like to express my appreciation to Dr. B. J. Sanderson. This work is part of a doctoral thesis written under his supervision and presented to the University of Warwick.

\section{EMBEDDINGS MODULO BORDISM}

In order to study the algebra $I(*, k)$ we have to consider first the $\mathscr{N}_{*}$-module $E(*, k)$ of bordism classes of embeddings. The definition of $E(n, k)$ is the same as that of $I(n, k)$ but now we take embeddings instead of immersions. The operations (a) and (c) give $E(*, k)$ the structure of an $\mathscr{N}_{*}$-module.

We denote by $M O$ the Thom spectrum for unoriented cobordism and by $\mathcal{N}_{*}(-)$ the associated geometric homology theory [6]. The structure of $E(*, k)$ is given by the following.

2.1 Theorem [17]. The Thom-Pontryagin construction defines an isomorphism of $\mathcal{N}_{*}$-modules $E(*, k) \cong \mathcal{N}_{*+k}\left(M O_{k}\right)$.

Recall that any map of spectra $\varphi: E \rightarrow F$ defines a natural transformation $\bar{\varphi}: E^{*}(-) \rightarrow F^{*}(-)$ between the cohomology theories defined by $E$ and $F$, and a natural transformation $\underline{\varphi}: E_{*}(-) \rightarrow F_{*}(-)$ between the associated homology theories. Using the fact that the smash product of spectra is a bifunctor on the homotopy category of spectra [14] one can easily prove the following. 
2.2 Lemma. Let $\varphi: E \rightarrow F$ be a map of ring spectra. (i) If $X$ is an $H$-space then $\underline{\varphi}: E_{*}(X) \rightarrow F_{*}(X)$ preserves the Pontryagin product. (ii) $\langle\bar{\varphi}(x), \underline{\varphi}(y)\rangle=$ $\underline{\varphi}\langle x, \bar{y}\rangle$, (iii) $\underline{\varphi}(a \cap b)=\bar{\varphi}(a) \cap \underline{\varphi}(b)$. (iv) $\bar{\varphi}$ and $\underline{\varphi}$ preserve the external products.

Let $H \mathbf{Z}_{2}$ be the Eilenberg-Mac Lane spectrum with $\mathbf{Z} / 2 \mathbf{Z} \equiv \mathbf{Z}_{2}$ coefficients. We denote by $t: M O \rightarrow H \mathrm{Z}_{2}$ the map of ring spectra defined by the universal Thom classes [3]. We have a natural transformation $\mu: \mathscr{N}_{*}(-) \rightarrow H_{*}\left(-; \mathbf{Z}_{2}\right)$ given by $\mu[M, f]=f_{*} \sigma(M)$, where $\sigma(M)$ is the fundamental class mod 2 of $M$. Thom proved that $\mu$ is surjective [15]. Let $M$ be a closed $n$-manifold. The fundamental class of $M$ in bordism $\stackrel{\circ}{\sigma}(M)$ is given by $\stackrel{\circ}{\sigma}(M)=[M$, id $] \epsilon$ $\mathscr{N}_{n}(M)$.

Using the fact that $\mu$ is the unique natural transformation that sends the fundamental class of a closed manifold in bordism to its fundamental class in homology and the properties in 2.2 , one can easily show that, under the natural equivalence $\mathscr{N}_{*}(-) \cong M O_{*}(-), \underline{t}$ corresponds to $\mu$. The structure of $\mathscr{N}_{*}(-)$ as an $\mathscr{N}_{*}$-module is given by the following.

2.3 Theorem [6]. Let $X$ be a space. The $\mathscr{N}_{*}$-module $\mathscr{N}_{*}(X)$ is free. A family $\left\{a_{i}\right\}_{i \in I}$ of homogeneous elements in $\mathscr{N}_{*}(X)$ is an $\mathscr{N}_{*}$-basis if and only if $\left\{\mu\left(a_{i}\right)\right\}_{i \in I}$ is a $\mathbf{Z}_{2}$-basis for $H_{*}\left(X ; \mathbf{Z}_{2}\right)$.

In order to find generators for the bordism of embeddings, we begin by giving generators for $\mathscr{N}_{*}(B O(k))$. Let $\xi$ be a vector bundle. We denote by $\stackrel{\circ}{e}(\xi)$ its Euler class and by $\stackrel{\circ}{t}(\xi)$ its Thom class in cobordism. We denote by $e(\xi)$ its Euler class and by $t(\xi)$ its Thom class in $\mathrm{Z}_{2}$-cohomology. Let $z: X \rightarrow T \xi$ be the inclusion of the zero section into the Thom space of $\xi$. Since $\stackrel{\circ}{e}(\xi)=$ $z^{*}(\stackrel{\circ}{t}(\xi))$ and $e(\xi)=z^{*}(t(\xi))$, it follows that $\bar{t}(\stackrel{\circ}{e}(\xi))=e(\xi)$. It is known [3]

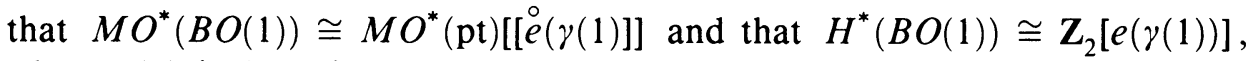
where $\gamma(1)$ is the universal bundle over $B O(1)$. We then have unique elements $\stackrel{\circ}{\beta}_{i} \in \widetilde{\mathcal{N}_{i}}(B O(1))$ and $\beta_{i} \in \widetilde{H}_{i}\left(B O(1) ; \mathbf{Z}_{2}\right)$ such that $\left\langle\stackrel{\circ}{e}(\gamma(1))^{i}, \stackrel{\circ}{\beta}_{j}\right\rangle=\delta_{i j}$ and $\left\langle e(\gamma(1))^{i}, \beta_{j}\right\rangle=\delta_{i j}$. We also define $\stackrel{\circ}{\beta}_{0}=[\{$ point $\}, l]$, where $l$ is the inclusion, and $\beta_{0}$ the generator of $H_{0}\left(B O(1) ; \mathbf{Z}_{2}\right)$.

Now we are going to give a basis for $H_{*}\left(B O(k) ; \mathbf{Z}_{2}\right)$. Take $\xi_{k}=\gamma(1) \times \cdots \times$ $\gamma(1)$ ( $k$ factors) with classifying map $m_{k}: B O(1)^{k} \rightarrow B O(k)$. The $i$ th StiefelWhitney class of $\xi_{k}$ is given by $m_{k}^{*}\left(w_{i}(\gamma(k))\right)=\sigma_{i}\left(u_{1}, u_{2}, \ldots, u_{k}\right)$, where $\sigma_{i}$ is the $i$ th elementary symmetric polynomial in $u_{1}, u_{2}, \ldots, u_{k}$ and where $u_{j} \in H^{1}\left(B O(1)^{k} ; \mathbf{Z}_{2}\right)$ is $p_{j}^{*}(e(\gamma(1))), p_{j}: B O(1)^{k} \rightarrow B O(1)$ the projection on the $j$ th factor. It is known that $m_{k}^{*}$ is an isomorphism onto the symmetric subalgebra $S \subset H^{*}\left(B O(1)^{k} ; \mathbf{Z}_{2}\right)$. For each sequence $0 \leq i_{1} \leq i_{2} \leq \cdots \leq i_{k}$, consider the monomial $u_{1}^{i_{1}} u_{2}^{i_{2}} \cdots u_{k}^{i_{k}}$ and let $\Phi_{i_{1}, i_{2}, \ldots, i_{k}}$ be the smallest symmetric polynomial containing this monomial. Clearly these polynomials are a basis for $S$. Therefore, for each collection $0 \leq i_{1} \leq i_{2} \leq \cdots \leq i_{k}$, the elements 
$\alpha_{i_{1}, i_{2}, \ldots, i_{k}} \in H^{*}\left(B O(k) ; \mathbf{Z}_{2}\right)$ such that $m_{k}^{*}\left(\alpha_{i_{1}, i_{2}, \ldots, i_{k}}\right)=\Phi_{i_{1}, i_{2}, \ldots, i_{k}}$, are a basis for $H^{*}\left(B O(k) ; \mathbf{Z}_{2}\right)$.

2.4 Lemma. Consider $\beta_{i} \in H_{i}\left(B O(1) ; \mathbf{Z}_{2}\right)$ and the map $m_{k}: B O(1)^{k} \rightarrow B O(k)$. The elements $m_{k_{*}}\left(\beta_{i_{1}} \times \beta_{i_{2}} \times \cdots \times \beta_{i_{k}}\right)$, for each collection $0 \leq i_{1} \leq i_{2} \leq \cdots \leq i_{k}$, form a basis for $H_{*}\left(B O(k) ; \mathbf{Z}_{2}\right)$.

Proof. It is easy to see that

$$
\left\{\alpha_{i_{1}, i_{2}, \ldots, i_{k}} \mid 0 \leq i_{1} \leq i_{2} \leq \cdots \leq i_{k}\right\}
$$

and

$$
\left\{m_{k_{*}}\left(\beta_{i_{1}} \times \beta_{i_{2}} \times \cdots \times \beta_{i_{k}}\right) \mid 0 \leq i_{1} \leq i_{2} \leq \cdots \leq i_{k}\right\}
$$

are dual bases.

2.5 Proposition. An $\mathscr{N}_{*}$-basis for $\mathscr{N}_{*}(B O(k))$ is given by the elements $m_{k_{*}}\left({\stackrel{\circ}{\beta_{i}}}_{i_{1}} \times{\stackrel{\circ}{\beta_{i}}}_{i_{2}} \times \cdots \times{\stackrel{\circ}{\beta_{i}}}_{i_{k}}\right)$, for each collection $0 \leq i_{1} \leq i_{2} \leq \cdots \leq i_{k}$.

Proof. Let us write $\stackrel{\circ}{e}$ for $\stackrel{\circ}{e}(\gamma(1))$ and $e$ for $e(\gamma(1))$. Using 2.2 we have $\left\langle e^{i}, \underline{t}\left(\stackrel{\circ}{\beta}_{j}\right)\right\rangle=\left\langle\bar{t}\left(\stackrel{\circ}{e}^{i}\right), \underline{t}\left(\stackrel{\circ}{\beta}_{j}\right)\right\rangle=\underline{t}\left\langle\stackrel{\circ}{e}^{i}, \stackrel{\circ}{\beta}_{j}\right\rangle=\underline{t}\left(\delta_{i j}\right)=\delta_{i j}$. Therefore $\underline{t}\left(\stackrel{\circ}{\beta}_{j}\right)=\beta_{j}$, and since we have identified $\underline{t}$ with $\mu$ it follows that $\mu\left(\stackrel{\circ}{\beta}_{j}\right)=\beta_{j}$. Using the naturality of $\mu$ and 2.2 we have that

$$
\mu m_{k_{*}}\left(\stackrel{\circ}{\beta}_{i_{1}} \times \stackrel{\circ}{\beta}_{i_{2}} \times \cdots \times \stackrel{\circ}{\beta}_{i_{k}}\right)=m_{k_{*}}\left(\beta_{i_{1}} \times \beta_{i_{2}} \times \cdots \times \beta_{i_{k}}\right) .
$$

The result now follows from 2.4 and 2.3.

2.6 Proposition. Consider the equivalence class of $\left(P^{n}, g_{n}\right)$ in $\mathscr{N}_{n}\left(P^{\infty}\right)$, where $g_{n}: P^{n} \rightarrow P^{\infty}$ is the inclusion $(n \geq 0)$. Then an $\mathscr{N}_{*}$-basis for $\mathscr{N}_{*}(B O(k))$ is given by the elements $m_{k_{*}}\left(\left[P^{i_{1}}, g_{i_{1}}\right] \times\left[P^{i_{2}}, g_{i_{2}}\right] \times \cdots \times\left[P^{i_{k}}, g_{i_{k}}\right]\right)$, for each collection $0 \leq i_{1} \leq i_{2} \leq \cdots \leq i_{k}$.

Proof. It is easy to see that

$$
\left\langle e(\gamma(1))^{i}, \mu\left[P^{j}, g_{j}\right]\right\rangle= \begin{cases}0 & \text { if } i \neq j \\ 1 & \text { if } i=j .\end{cases}
$$

Therefore $\mu\left[P^{n}, g_{n}\right]=\beta_{n}$ and the result follows from 2.4 and 2.3.

We now give specific representatives for the elements $\stackrel{\circ}{\beta}_{i} \in \widetilde{\mathcal{N}_{i}}(B O(1))$.

2.7 Definition. We define $b_{k} \in \mathcal{N}_{k}\left(P^{\infty}\right)$ to be the equivalence class of $H(1, k)$ $\subset P^{1} \times P^{k} \stackrel{\pi}{\rightarrow} P^{k} \stackrel{g_{k}}{\rightarrow} P^{\infty}$ where $H(1, k)$ is the Milnor manifold [11] (a hypersurface contained in $P^{1} \times P^{k}$ ) and $\pi$ is the projection.

2.8 Lemma. $\stackrel{\circ}{e}(\gamma(1)) \cap b_{k}=b_{k-1}$.

Proof. Let $\gamma_{m}$ be the restriction of $\gamma(1)$ to $P^{m}$ and let $j_{k}: P^{k-1} \rightarrow P^{k}$ be the inclusion. We denote the equivalence class of $H(1, k) \rightarrow P^{1} \times P^{k}$ by $a_{k}$. Then 
$\stackrel{\circ}{e}(\gamma(1)) \cap b_{k}=\left(g_{k} \circ \pi\right)_{*}\left(1 \times \stackrel{\circ}{e}\left(\gamma_{k}\right) \cap a_{k}\right)$. On the other hand

$$
b_{k-1}=\left(g_{k} \circ \pi\right)_{*}\left(\stackrel{\circ}{e}\left(\gamma_{1} \otimes \gamma_{k}\right) \cap\left(\operatorname{id} \times j_{k}\right)_{*}\left(\sigma\left(P^{1} \times P^{k-1}\right)\right)\right) \text {. }
$$

But $\stackrel{\circ}{e}\left(\gamma_{k}\right)$ is Poincaré dual to $\left[P^{k-1}, j_{k}\right]$ and so $1 \times \stackrel{\circ}{e}\left(\gamma_{k}\right)$ is Poincaré dual to $\left(\text { id } \times j_{k}\right)_{*} \sigma\left(P^{1} \times P^{k-1}\right)$. It is known that $a_{k}$ is Poincaré dual to $\stackrel{\circ}{e}\left(\gamma_{1} \otimes \gamma_{k}\right)$ [3]. Therefore their cap products are the same and the lemma follows.

2.9 Proposition. An $\mathscr{N}_{*}$-basis for $\mathscr{N}_{*}(B O(k))$ is given by the elements $m_{k_{*}}\left(b_{i_{1}} \times\right.$ $\left.b_{i_{2}} \times \cdots \times b_{i_{k}}\right)$, for each collection $0 \leq i_{1} \leq i_{2} \leq \cdots \leq i_{k}$.

Proof. We denote by $P(\xi)$ the projective bundle associated to a vector bundle $\xi$. Then $H(1, n) \cong P\left(\gamma_{1} \oplus \varepsilon^{n-1}\right)$, where $\varepsilon^{n-1}$ is the trivial $(n-1)$-bundle. Hence by [7] $[H(1, n)]=0$ in $\mathscr{N}_{n}(n \geq 1)$. With this result and 2.8 we have that

$$
\begin{aligned}
\left\langle\stackrel{\circ}{e}(\gamma(1))^{j}, b_{k}\right\rangle & =\left\langle 1, \stackrel{\circ}{e}(\gamma(1)) \cap \cdots \cap \stackrel{\circ}{e}(\gamma(1)) \cap b_{k}\right\rangle=\left\langle 1, b_{k-j}\right\rangle \\
& = \begin{cases}\left\langle 1, b_{0}\right\rangle=1 & \text { if } k=j, \\
\left\langle 1, b_{k-j}\right\rangle=[H(1, k-j)]=0 & \text { if } k \neq j .\end{cases}
\end{aligned}
$$

Therefore $b_{k}=\stackrel{\circ}{\beta}_{k}$ and the result follows from 2.5.

2.10 Remark. In order to give the generators for $E(*, k)$ we define the following isomorphisms.

Let $f:(M, \partial M) \rightarrow\left(X, x_{0}\right)$ be a map of pairs from a smooth manifold with boundary to a pointed space. Let $\widetilde{M}$ be the double of $M$ and let $\tilde{f}: \widetilde{M} \rightarrow X$ be the map induced by $f \amalg c: M \amalg M \rightarrow X$, where $c(y)=x_{0}$ for all $y \in M$. We define $d: \mathscr{N}_{n}\left(X, x_{0}\right) \rightarrow \mathscr{N}_{n}(X)$ by $d[M, f]=[\widetilde{M}, \tilde{f}]$. Let $i:\left\{x_{0}\right\} \rightarrow X$ denote the inclusion. One can easily show that $\Psi: \mathscr{N}_{n} \oplus \mathscr{N}_{n}\left(X, x_{0}\right) \rightarrow \mathscr{N}_{n}(X)$ given by $\Psi(a, b)=i_{*}(a)+d(b)$, is an isomorphism.

Now let $\xi=(E, p, V)$ be a smooth $k$-vector bundle. We define $\phi: \mathscr{N}_{n}(V) \rightarrow$ $\mathscr{N}_{n+k}(T \xi, *)$ as follows. Take $[N, g] \in \mathscr{N}_{n}(V)$ and consider the pull-back $g^{*}(\xi)$. If we give a Riemannian metric to $\xi$ and we put the pull-back metric on $g^{*}(\xi)$ then we get a map $\dot{g}:\left(D\left(g^{*} \xi\right), S\left(g^{*} \xi\right)\right) \rightarrow(D(\xi), S(\xi))$, where $D(-)$ denotes the disc bundle and $S(-)$ the sphere bundle. If $q:(D(\xi), S(\xi)) \rightarrow(T \xi, *)$ denotes the quotient map, then $\phi$ is given by $\phi[N, g]=[D(g * \xi), q \circ \dot{g}]$. From the geometric definition of the Thom isomorphism $\psi$ given in [3] one can see that $\phi=\psi^{-1}$. Hence $\phi$ is an isomorphism.

2.11 Definition. Let $H(1, n) \subset P^{1} \times P^{n}$ be the Milnor manifold. We denote by $\mathscr{H}_{n}$ the restriction to $H(1, n)$ of the line bundle $P^{1} \times \gamma_{n} \rightarrow P^{1} \times P^{n}$. For each collection $0 \leq i_{1} \leq i_{2} \leq \cdots \leq i_{k}$ consider $\widetilde{D}\left(\mathscr{H}_{i_{1}} \times \mathscr{H}_{i_{2}} \times \cdots \times \mathscr{H}_{i_{k}}\right)$, the double of the disc bundle, and the embedding $H\left(1, i_{1}\right) \times H\left(1, i_{2}\right) \times \cdots \times H\left(1, i_{k}\right) \rightarrow$ $\widetilde{D}\left(\mathscr{H}_{i_{1}} \times \mathscr{H}_{i_{2}} \times \cdots \times \mathscr{H}_{i_{k}}\right)$ as the zero section. Similarly we construct the embedding

$$
P^{i_{1}} \times P^{i_{2}} \times \cdots \times P^{i_{k}} \rightarrow \widetilde{D}\left(\gamma_{i_{1}} \times \gamma_{i_{2}} \times \cdots \times \gamma_{i_{k}}\right) .
$$


We also consider the empty collection and we associate to this one the embedding $\phi \subset\{$ point $\}$. With this notation we have the following.

2.12 Theorem. (a) The embeddings

$$
H\left(1, i_{1}\right) \times H\left(1, i_{2}\right) \times \cdots \times H\left(1, i_{k}\right) \rightarrow \widetilde{D}\left(\mathscr{H}_{i_{1}} \times \mathscr{H}_{i_{2}} \times \cdots \times \mathscr{H}_{i_{k}}\right),
$$

for each collection $0 \leq i_{1} \leq i_{2} \leq \cdots \leq i_{k}$, are an $\mathscr{N}_{*}$-basis for $E(*, k)$.

(b) The embeddings

$$
P^{i_{1}} \times P^{i_{2}} \times \cdots \times P^{i_{k}} \rightarrow \widetilde{D}\left(\gamma_{i_{1}} \times \gamma_{i_{2}} \times \cdots \times \gamma_{i_{k}}\right),
$$

for each collection $0 \leq i_{1} \leq i_{2} \leq \cdots \leq i_{k}$, are an $\mathscr{N}_{*}$-basis for $E(*, k)$.

Proof. The theorem follows from 2.1, 2.10, 2.9 and 2.6.

\section{DYER-LASHOF OPERATIONS IN HOMOLOGY AND IN BORDISM}

We begin this section with the theorem that gives an isomorphism between the bordism algebra of immersions and the bordism of an infinite loop space. Given a pointed space $\left(X, x_{0}\right)$, we denote by $Q X$ the direct limit, under suspensions, $\lim _{n} \Omega^{n} S^{n}\left(X, x_{0}\right)$, where $\Omega^{n}(-)$ denotes the space of $n$-loops and where $S^{n}(-)$ denotes the $n$th suspension.

3.1 Definition. We define $\alpha_{k}: I(n, k) \rightarrow \mathcal{N}_{n+k}\left(Q M O_{k}\right)$ as follows. Let $[f: M \rightarrow N] \in I(n, k)$. We can find an embedding $f_{0}: M \rightarrow N \times \mathbf{R}^{r}$ (taking $r>n-k+1)$ such that $f_{0}$ is regularly homotopic to the composition $M \stackrel{f}{\rightarrow} N \subset N \times \mathbf{R}^{r}$. Clearly we have that $\nu_{f_{0}} \cong \nu_{f} \oplus \varepsilon^{r}$, where $\nu$ denotes the normal bundle. Applying the Thom-Pontryagin construction we get a map $t_{f}: S^{r}\left(N^{+}\right) \rightarrow S^{r}\left(T\left(\nu_{f}\right)\right)$, where $N^{+}$denotes $N \amalg\{+\}$. The classifying map for $\nu_{f}, M \rightarrow B O(k)$ induces a map of Thom spaces $\tau_{f}: T\left(\nu_{f}\right) \rightarrow M O_{k}$. We can take the composition

$$
S^{r}\left(N^{+}\right) \stackrel{t_{f}}{\rightarrow} S^{r}\left(T \nu_{f}\right) \stackrel{S^{r} \tau_{f}}{\rightarrow} S^{r} M O_{k} .
$$

Taking the adjoint of this map we get a map $N \subset N^{+} \rightarrow \Omega^{r} S^{r} M O_{k} \subset Q M O_{k}$. Then $\alpha_{k}[f: M \rightarrow N] \in \mathcal{N}_{n+k}\left(Q M O_{k}\right)$ is the equivalence class of this map. With this notation we have the following

3.2 Theorem [13]. $\alpha_{k}: I(*, k) \rightarrow \mathcal{N}_{*+k}\left(Q M O_{k}\right), k>0$, is an isomorphism of $\mathscr{N}_{*}$-algebras.

In order to calculate $\mathcal{N}_{*}\left(Q M O_{k}\right)$ we need the Dyer-Lashof operations. To define the operations we need the following two propositions [8]. Let $E$ be a contractible space with a free action of $\Sigma_{2}$ and let $\Sigma_{2}$ act on $X^{2}$ by permuting the factors. We denote by $B_{*}$ the normalized Bar resolution for $Z_{2}$ over $\Sigma_{2}$ (hence each $B_{n}$ is a free $\mathbf{Z}_{2}\left[\Sigma_{2}\right]$-module in one generator $e_{n}$ ). 
3.3 Proposition. There is a natural isomorphism

$$
H_{*}\left(E \times_{\Sigma_{2}} X^{2} ; \mathbf{Z}_{2}\right) \cong H_{*}\left(B_{*} \otimes_{\mathbf{Z}_{2}\left[\Sigma_{2}\right]} H_{*}(X)^{\otimes 2}\right),
$$

where $H_{*}(X)^{\otimes 2}$ is a chain complex with trivial boundary and with $\Sigma_{2}$ acting by permuting the coordinates.

3.4 Proposition. Let $\left\{a_{j}\right\}_{j \in J}$ be an ordered basis for $H_{*}\left(X ; \mathbf{Z}_{2}\right) . A \mathbf{Z}_{2}$-basis for $H_{*}\left(B_{*} \otimes_{\mathbf{Z}_{2}\left[\Sigma_{2}\right]} H_{*}(X)^{\otimes 2}\right)$ is given by the following elements:

$$
\left\{\begin{array}{l}
e_{r} \otimes_{\Sigma_{2}} a_{j} \otimes a_{j}, \quad r \geq 0, j \in J, \\
e_{0} \otimes_{\Sigma_{2}} a_{j} \otimes a_{k}, \quad j<k .
\end{array}\right.
$$

3.5 Definition [4, 5, 8]: Let $\mathscr{C}$ be an $E_{\infty}$-operad and $X$ a $\mathscr{C}$-space [10] with structure maps $\theta_{r}: \mathscr{C}(r) \times_{\Sigma_{r}} X^{r} \rightarrow X$. We have homomorphisms $Q_{i}: H_{n}(X) \rightarrow$ $H_{2 n+i}(X)$, which are natural with respect to maps of $\mathscr{C}$-spaces, defined by the following composition:

$$
\begin{aligned}
& H_{n}\left(X ; \mathbf{Z}_{2}\right) \stackrel{h_{i}^{\infty}}{\rightarrow} H_{2 n+i}\left(B_{*} \otimes_{\mathbf{Z}_{2}\left[\Sigma_{2}\right]} H_{*}(X)^{\otimes 2}\right) \stackrel{\cong}{\cong} H_{2 n+i}\left(\mathscr{C}(2) \times_{\Sigma_{2}} X^{2} ; \mathbf{Z}_{2}\right) \\
& \quad \stackrel{\theta_{2^{*}}}{\longrightarrow} H_{2 n+i}\left(X ; \mathbf{Z}_{2}\right)
\end{aligned}
$$

where $h_{i}^{\infty}(a)=e_{i} \otimes_{\Sigma_{2}} a \otimes a$.

In particular if $X$ is an infinite loop space then $X$ is a $\mathscr{C}_{\infty}$-space, where $\mathscr{C}_{\infty}$ is the cubes operad. Since $\mathscr{C}_{\infty}$ is an $E_{\infty}$-operad we have Dyer-Lashof operations in the homology of $X$.

Now following $[2,12,16]$ we define Dyer-Lashof operations in the bordism of a $\mathscr{C}_{\infty}$-space. We begin by defining functions

$$
\tilde{q}_{r}^{n}: \mathscr{N}_{s}(X) \rightarrow \mathscr{N}_{2 s+r}\left(S^{n} \times_{\Sigma_{2}} X \times X\right), \quad r \leq n \leq \infty,
$$

as follows. If $[M, f] \in N_{s}(X)$, then $\tilde{q}_{r}^{n}[M, f]=\left[S^{r} \times{ }_{\Sigma_{2}} M^{2}, i \times_{\Sigma_{2}} f^{2}\right]$. (Here $\Sigma_{2}$ acts on $S^{r}$ by the antipodal action and on $M^{2}$ by permuting the coordinates; $i: S^{r} \rightarrow S^{n}$ is the usual inclusion.) It is easy to see that when $r<n, \tilde{q}_{r}^{n}$ is a homomorphism.

3.6 Definition. Let $X$ be a $\mathscr{C}_{\infty}$-space. We define homomorphisms $\widetilde{Q}_{r}: \mathscr{N}_{n}(X)$ $\rightarrow \mathscr{N}_{2 n+r}(X)$, which are natural with respect to maps of $\mathscr{C}_{\infty}$-spaces, as follows. There is a $\Sigma_{2}$-equivariant homotopy equivalence $j: S^{\infty} \rightarrow \mathscr{C}_{\infty}$ (2) [10] so we have a homotopy equivalence $S^{\infty} \times_{\Sigma_{2}} X \times X \simeq \mathscr{C}_{\infty}(2) \times_{\Sigma_{2}} X \times X . \widetilde{Q}_{r}$ is the composition:

$$
\begin{aligned}
\mathscr{N}_{n}(X) & \stackrel{\dot{q}_{r}^{\infty}}{\rightarrow} \mathscr{N}_{2 n+r}\left(S^{\infty} \times_{\Sigma_{2}} X \times X\right) \\
& \cong \mathscr{N}_{2 n+r}\left(\mathscr{C}_{\infty}(2) \times_{\Sigma_{2}} X \times X\right) \stackrel{\theta_{2 *}}{\rightarrow} \mathscr{N}_{2 n+r}(X) .
\end{aligned}
$$

The operations $\tilde{q}_{r}^{n}$ were defined when $X$ is a closed manifold in [16]. The operations $\widetilde{Q}_{r}$ satisfy properties analogous to those satisfied by the Dyer-Lashof operations in homology [2, 12]. 
Now we are going to see that the operations $\widetilde{Q}_{r}$ correspond under $\mu$ to the operations $Q_{r}$. Let us denote by $B_{*}^{(n)}$ the $n$-skeleton of the normalized Bar resolution. We have, as in 3.3, a natural isomorphism

$$
H_{*}\left(S^{n} \times_{\Sigma_{2}} X \times X ; \mathbf{Z}_{2}\right) \cong H_{*}\left(B_{*}^{(n)} \otimes_{\mathbf{Z}_{2}\left[\Sigma_{2}\right]} H_{*}(X)^{\otimes 2}\right) .
$$

Consequently, we can define natural functions

$$
q_{r}^{n}: H_{s}\left(X ; \mathbf{Z}_{2}\right) \rightarrow H_{2 s+r}\left(S^{n} \times_{\Sigma_{2}} X \times X ; \mathbf{Z}_{2}\right), \quad 0 \leq r \leq n \leq \infty,
$$

by the composition

$$
H_{s}\left(X ; \mathbf{Z}_{2}\right) \stackrel{h_{r}^{n}}{\rightarrow} H_{2 s+r}\left(B_{*}^{(n)} \times_{\mathbf{Z}_{2}\left[\Sigma_{2}\right]} H_{*}(X)^{\otimes 2}\right) \stackrel{\cong}{\rightrightarrows} H_{2 s+r}\left(S^{n} \times_{\Sigma_{2}} X \times X ; \mathbf{Z}_{2}\right),
$$

where $h_{r}^{n}(a)=e_{r} \otimes_{\Sigma_{2}} a \otimes a$.

3.7 Lemma. Suppose $M$ is a connected m-manifold with fundamental class $\sigma(M)$. We have $\sigma\left(S^{r} \times \Sigma_{\Sigma_{2}} M \times M\right)=q_{r}^{r}(\sigma(M))$.

Proof. Consider $q_{r}^{r}: H_{m}\left(M ; \mathbf{Z}_{2}\right) \rightarrow H_{2 m+r}\left(S^{r} \times \Sigma_{\Sigma_{2}} M \times M ; \mathbf{Z}_{2}\right)$. As $M$ is connected, $S^{r} \times_{\Sigma_{2}} M \times M$ is also connected. Hence $H_{2 m+r}\left(S^{r} \times \Sigma_{\Sigma_{2}} M \times M ; \mathbf{Z}_{2}\right) \cong \mathbf{Z}_{2}$. Consider the inclusion $i \times \Sigma_{\Sigma_{2}}$ id: $S^{r} \times \Sigma_{\Sigma_{2}} M \times M \rightarrow S^{\infty} \times{ }_{\Sigma_{2}} M \times M$. It is easy to see that $\left(i \times_{\Sigma_{2}} \text { id }\right)_{*} \circ q_{r}^{r}=q_{r}^{\infty}$. Hence $\left(i \times_{\Sigma_{2}} \text { id }\right)_{*} q_{r}^{r}(\sigma(M))=q_{r}^{\infty}\left(\sigma_{M}\right) \neq 0$ by 3.4, and so $q_{r}^{r}(\sigma(M))=\sigma\left(S^{r} \times \Sigma_{2} M \times M\right)$.

3.8 Lemma. The following diagram commutes:

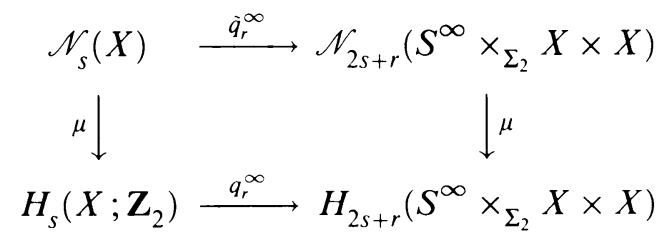

Proof. The elements $[M, f] \in N_{s}(X)$ with $M$ connected generate so we can assume that $M$ is connected. Then

$$
\mu \tilde{q}_{r}^{\infty}[M, f]=\left(i \times_{\Sigma_{2}} f \times f\right)_{*} \sigma\left(S^{r} \times_{\Sigma_{2}} M \times M\right)=\left(i \times_{\Sigma_{2}} f \times f\right)_{*} q_{r}^{r}(\sigma(M))
$$

by 3.7. But

and

$$
i \times_{\Sigma_{2}} f \times f=\mathrm{id} \times_{\Sigma_{2}} f \times f \circ i \times_{\Sigma_{2}} \mathrm{id}
$$

$$
\left(i \times_{\Sigma_{2}} \mathrm{id}\right)_{*} \circ q_{r}^{r}=q_{r}^{\infty} .
$$

By the naturality of $q_{r}^{\infty}$, we get

$$
\left(i \times \Sigma_{\Sigma_{2}} f \times f\right)_{*} q_{r}^{r}(\sigma(M))=q_{r}^{\infty} f_{*}(\sigma(M))=q_{r}^{\infty} \mu[M, f] .
$$

3.9 Proposition. Let $X$ be a $\mathscr{C}_{\infty}$-space. The following diagram commutes:

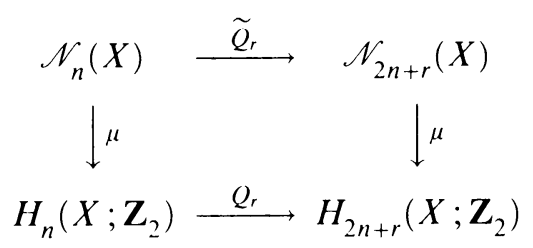


Proof. Consider the following diagram:

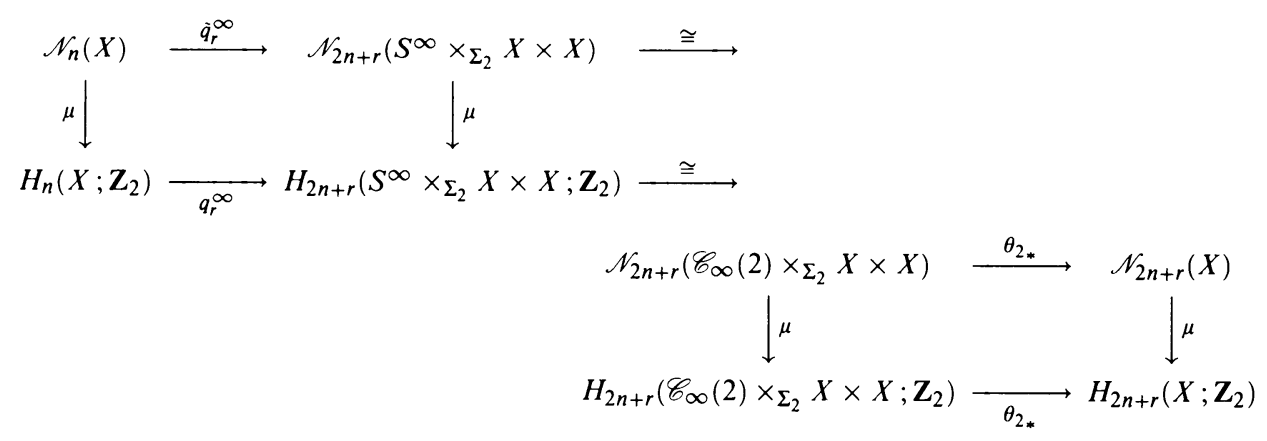

The first square commutes by 3.8 , the other two by naturality of $\mu$. The composition at the top is the definition of $\widetilde{Q}_{r}$. It is easy to see that the composition at the bottom coincides with $Q_{r}$ using the generalization of the theorem of acyclic models due to Dyer and Lashof [8].

With these operations we can give the structure of $H_{*}\left(Q X ; \mathbf{Z}_{2}\right)$ and $\mathscr{N}_{*}(Q X)$ as follows: consider sequences $I=\left(i_{1}, i_{2}, \ldots, i_{d}\right)$ such that $0<i_{1} \leq i_{2} \leq$ $\cdots \leq i_{d}, d \geq 0$, and call these sequences monotone. Take the iterated product $Q_{I}=Q_{i_{1}} Q_{i_{2}} \cdots Q_{i_{d}}$ and $\widetilde{Q}_{I}=\widetilde{Q}_{i_{1}} \widetilde{Q}_{i_{2}} \cdots \widetilde{Q}_{i_{d}}$. We have the following

3.10 Theorem $[4,5,8]$. Let $X$ be a connected space and let $\left\{X_{\alpha}\right\}_{\alpha \in \Lambda}$ be a $\mathbf{Z}_{2^{-}}$ basis for $\widetilde{H}_{*}\left(X ; \mathbf{Z}_{2}\right) \subset H_{*}\left(Q X ; \mathbf{Z}_{2}\right)$. There is an isomorphism of $\mathbf{Z}_{2}$-algebras $H_{*}\left(Q X ; \mathbf{Z}_{2}\right) \cong \mathbf{Z}_{2}\left[Q_{I}\left(x_{*}\right) \mid I\right.$ is monotone, and $\left.\alpha \in \Lambda\right]$.

3.11 Proposition. Let $X$ be a connected space and let $\left\{y_{\alpha}\right\}_{\alpha \in \Lambda}$ be an $\mathscr{N}_{*}$-basis for $\widetilde{\mathscr{N}_{*}}(X) \subset \mathscr{N}_{*}(Q X)$. There is an isomorphism of $\mathscr{N}_{*}$-algebras $\mathscr{N}_{*}(Q X) \cong$ $\mathscr{N}_{*}\left[\widetilde{Q}_{I}\left(y_{\alpha}\right) \mid I\right.$ is monotone and $\left.\alpha \in \Lambda\right]$.

Proof. Consider a monomial $\widetilde{Q}_{I_{1}}\left(y_{\alpha_{1}}\right)^{r_{1}} \widetilde{Q}_{I_{2}}\left(y_{\alpha_{2}}\right)^{r_{2}} \cdots \widetilde{Q}_{I}\left(y_{\alpha_{m}}\right)^{r_{m}}$, where each $I_{s}$ is monotone. By 2.2 and 3.9 we have

$$
\begin{aligned}
& \mu\left(\widetilde{Q}_{I_{1}}\left(y_{\alpha_{1}}\right)^{r_{1}} \widetilde{Q}_{I_{2}}\left(y_{r_{2}}\right)^{r_{2}} \cdots \widetilde{Q}_{I_{m}}\left(y_{\alpha_{m}}\right)^{r_{m}}\right) \\
& \quad=Q_{I_{1}}\left(\mu\left(y_{\alpha_{1}}\right)\right)^{r_{1}} Q_{I_{2}}\left(\mu\left(y_{\alpha_{2}}\right)\right)^{r_{2}} \cdots Q_{I_{m}}\left(\mu\left(y_{\alpha_{m}}\right)\right)^{r_{m}} .
\end{aligned}
$$

Since $\left\{y_{\alpha}\right\}_{r \in \Lambda}$ is an $\mathscr{N}_{*}$-basis for $\widetilde{\mathscr{N}}(X)$ then, by Theorem 2.3, the elements $\left\{\mu\left(y_{n}\right)\right\}_{k \in \Lambda}$ are a $\mathbf{Z}_{2}$-basis for $\widetilde{H}_{*}\left(X ; \mathbf{Z}_{2}\right)$. Hence by Theorem 3.10 the elements $\mu\left(\widetilde{Q}_{I_{1}}\left(y_{r_{1}}\right)^{r_{1}} \tilde{Q}_{I_{2}}\left(y_{r_{2}}\right)^{r_{2}} \ldots \widetilde{Q}_{I_{m}}\left(y_{r_{m}}\right)^{r_{m}}\right)$ are a $\mathbf{Z}_{2}$-basis for $H_{*}\left(Q X ; \mathbf{Z}_{2}\right)$. The proposition follows from 2.3. 
4. THE OPERATIONS $\widehat{Q}_{r}: I(n, k) \rightarrow I(2 n+k+r, k)$

4.1 Definition. We define operations $\widehat{Q}_{r}: I(n, k) \rightarrow I(2 n+k+r, k), k>0$, by the commutativity of the following diagram:

$$
\begin{array}{cc}
I(n, k) \stackrel{\widehat{Q}_{r}}{\longrightarrow} I(2 n+k+r, k) \\
{ }_{\alpha_{k}} \cong & \cong \downarrow \alpha_{k} \\
\mathscr{N}_{n+k}\left(Q M O_{k}\right) \stackrel{\widetilde{Q}_{r}}{\longrightarrow} \mathscr{N}_{2(n+k)+r}\left(Q M O_{k}\right)
\end{array}
$$

We will give a description of $\widehat{Q}_{r}$. In order to do this we need some preliminary results.

The following two lemmas can be easily proved.

4.2 Lemma. Let $f: M \rightarrow \check{N}$ be an immersion and let $p: \check{N} \rightarrow N$ be a covering space. Consider the maps associated to $f$ and to $p$ by the Thom-Pontryagin construction $t_{f}: S^{\infty}\left(\check{N}^{+}\right) \rightarrow S^{\infty}\left(T \nu_{f}\right)$ and $t_{p}: S^{\infty}\left(N^{+}\right) \rightarrow S^{\infty}\left(\check{N}^{+}\right)$, where $S^{\infty}(-)$ denotes the suspension spectrum. Then the following diagram is homotopy commutative:

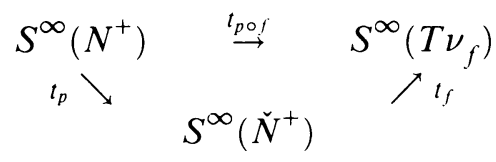

4.3 Lemma. Let $f: M \rightarrow N$ be an immersion and $V$ a manifold. Consider the immersion id $\times f: V \times M \rightarrow V \times N$. If the Thom-Pontryagin map of $f$ is given by $S^{\infty}\left(N^{+}\right) \stackrel{t_{f}}{\rightarrow} S^{\infty}\left(T \nu_{f}\right) \stackrel{S^{\infty} \tau_{f}}{\rightarrow} S^{\infty} M O_{k}$ then the Thom-Pontryagin map of $\mathrm{id} \times f$ is given by

$$
V^{+} \wedge S^{\infty}\left(N^{+}\right) \stackrel{\text { id } \wedge t_{f}}{\rightarrow} V^{+} \wedge S^{\infty}\left(T \nu_{f}\right) \stackrel{\pi}{\rightarrow} S^{\infty}\left(T \nu_{f}\right) \stackrel{S^{\infty} \tau_{f}}{\rightarrow} S^{\infty} M O_{k}
$$

where $\pi$ is induced by the projection on the second factor.

We now recall the definition of the Kahn-Priddy transfer for covering spaces $[1,9]$. Let $p: X \rightarrow Y$ be an $n$-covering ( $X, Y$ C.W.-complexes) and take $\bar{X}=\left\{\left(x_{1}, x_{2}, \ldots, x_{n}\right) \in X^{n} \mid x_{i} \neq x_{j}\right.$ if $i \neq j$ and $\left.p\left(x_{1}\right)=p\left(x_{2}\right)=\cdots=p\left(x_{n}\right)\right\}$. Then $\bar{X}$ is a free $\Sigma_{n}$-space and the projection $\bar{X} \rightarrow \bar{X} / \Sigma_{n}$ is the principal $\Sigma_{n}$ bundle associated to $p$. Let $\lambda: \bar{X} \rightarrow \mathscr{C}_{\infty}(n)$ be a classifying map (unique up to equivariant homotopy). We define a map $\phi: Y \cong \bar{X} / \Sigma_{n} \rightarrow \mathscr{C}_{\infty}(n) \times_{\Sigma_{n}} X^{n}$ by

$$
\phi\left[x_{1}, x_{2}, \ldots, x_{n}\right]=\left[\lambda\left(x_{1}, x_{2}, \ldots, x_{n}\right),\left(x_{1}, x_{2}, \ldots, x_{n}\right)\right]
$$

and we call the unique map extending $\phi, T: Y^{+} \rightarrow \mathscr{C}_{\infty}(n) \times_{\Sigma_{n}}\left(X^{+}\right)^{n}$ the pretransfer. Let $i: X^{+} \rightarrow Q\left(X^{+}\right)$be the inclusion. The following composition, denoted by $\tau(p)$, is the transfer for $p$ :

$$
Y^{+} \stackrel{T}{\rightarrow} \mathscr{C}_{\infty}(n) \times_{\Sigma_{n}}\left(X^{+}\right)^{n} \underset{\text { id } \times_{\Sigma_{n}} i^{n}}{\rightarrow} \mathscr{C}_{\infty}(n) \times_{\Sigma_{n}}\left(Q\left(X^{+}\right)\right)^{n} \underset{\theta_{n}}{\rightarrow} Q\left(X^{+}\right) .
$$


4.4 Proposition [1]. Let $p: \check{N} \rightarrow N$ be an $n$-covering, where $\check{N}$ and $N$ are closed smooth manifolds. The adjoint of the Thom-Pontryagin map associated to $p, \operatorname{adj} t_{p}: N \rightarrow Q\left(\check{N}^{+}\right)$and the transfer for $p, \tau(p): N \rightarrow Q\left(\check{N}^{+}\right)$are homotopic.

4.5 Theorem. The operations $\widehat{Q}_{r}: I(n, k) \rightarrow I(2 n+k+r, k), k>0$, are given by $\hat{Q}_{r}[f: M \rightarrow N]=\left[\hat{f}: S^{r} \times N \times M \rightarrow S^{r} \times_{\Sigma_{2}} N \times N\right]$, where $\hat{f}(a, y, x)=$ $[a, y, f(x)]$.

Proof. We can write $\hat{f}$ as the composition:

$$
S^{r} \times N \times M \underset{\operatorname{id}_{S^{r} \times N} \times f}{\rightarrow} S^{r} \times N \times N \underset{p}{\rightarrow} S^{r} \times \Sigma_{\Sigma_{2}} N \times N .
$$

By 3.1 the element of $\mathscr{N}_{n+k}\left(Q M O_{k}\right)$ associated to $f$ is given by the adjoint of $S^{\infty}\left(N^{+}\right) \stackrel{t_{f}}{\rightarrow} S^{\infty}\left(T \nu_{f}\right) \stackrel{S^{\infty} \tau_{f}}{\rightarrow} S^{\infty} M O_{k}$. Let $\phi=\operatorname{adj}\left(S^{\infty} \tau_{f} \circ t_{f}\right): N \rightarrow Q M O_{k}$ then $\widetilde{Q}_{r}[N, \phi]$ is given by the composition:

$$
\begin{aligned}
& S^{r} \times_{\Sigma_{2}} N \times N \underset{i \times \Sigma_{2} \phi \times \phi}{\rightarrow} S^{\infty} \times_{\Sigma_{2}} Q M O_{k} \times Q M O_{k} \\
& \underset{j \times \Sigma_{2} \mathrm{id} \times \text { id }}{\simeq} \mathscr{C}_{\infty}(2) \times_{\Sigma_{2}} Q M O_{k} \times Q M O_{k} \underset{\theta_{2}}{\rightarrow} Q M O_{k} .
\end{aligned}
$$

By 4.2 and 4.3 we have the following homotopy commutative diagram

$$
\begin{array}{ccc}
S^{\infty}\left[\left(S^{r} \times \Sigma_{\Sigma_{2}} N \times N\right)^{+}\right] & \stackrel{t_{f}}{\longrightarrow} & S^{\infty}\left(T \nu_{f}\right) \stackrel{S^{\infty} \tau_{f}}{\rightarrow} S^{\infty} M O_{k} \\
S^{\infty}\left[\left(S^{r} \times N \times N\right)^{+}\right] & & S^{\infty}\left[\left(S^{r} \times N\right)^{+} \wedge T \nu_{f}\right] \\
\left(S^{r} \times N\right)^{+} \wedge S^{\infty}\left(N^{+}\right) & \underset{\mathrm{id} \wedge t_{f}}{\longrightarrow} & \left(S^{r} \times N\right)^{+} \wedge S^{\infty} T \nu_{f}
\end{array}
$$

To prove the theorem we are going to show that (1) and the adjoint of (2) are homotopic, thus representing the same element in $\mathscr{N}_{2(n+k)+r}\left(Q M O_{k}\right)$. To do this we construct a transfer for the covering $S^{r} \times N \times N \stackrel{p}{\rightarrow} S^{r} \times \Sigma_{\Sigma_{2}} N \times N$ as follows. Consider the following diagram:

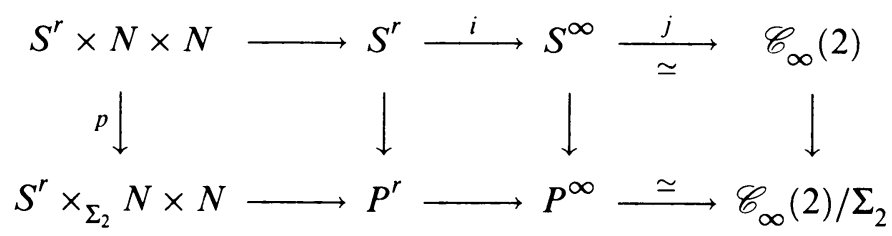

The first square is induced by the projection. The composition at the top is equivariant so we have a pull-back diagram. We then have a pretransfer $T$ which in this case has the form $T\left(a, y_{1}, y_{2}\right)=\left[j \circ i(a),\left(-a, y_{2}, y_{1}\right),\left(a, y_{1}, y_{2}\right)\right]$. This gives a transfer for $p$ that we denote by $\tau(p)$.

Let $\Omega^{\infty}$ be the functor that associates to each spectrum the 0th space of the associated $\Omega$-spectrum. 
The map associated to the immersion $\hat{Q}_{r}[f: M \rightarrow N]$ is given by the adjoint of (2) so, by the naturality of adjointness, it is homotopic to $Q \tau_{f} \circ Q \pi \circ$ $\Omega^{\infty}\left(\operatorname{id} \wedge t_{f}\right) \circ \operatorname{adj} t_{p}$. By $4.4 \operatorname{adj} t_{p}$ is homotopic to the transfer for $p$. In particular $\operatorname{adj} t_{p} \simeq \tau(p)$. Hence the map associated to $\widehat{Q}_{p}[M, f]$ is homotopic to

$$
Q \tau_{f} \circ Q \pi \circ \Omega^{\infty}\left(\mathrm{id} \wedge t_{f}\right) \circ \tau(p) .
$$

Now putting side by side the two diagrams below we see that the composition at the bottom is (3). The triangle commutes by definition of the transfer and the squares commute because all the maps are infinite loop maps. Therefore the composition at the bottom is the same as the composition at the top, which, as one can easily verify, is precisely (1).

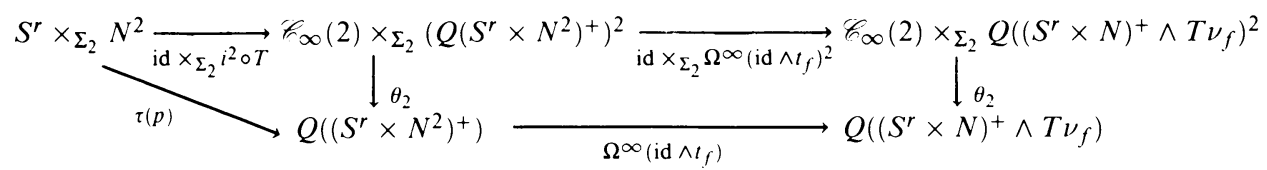

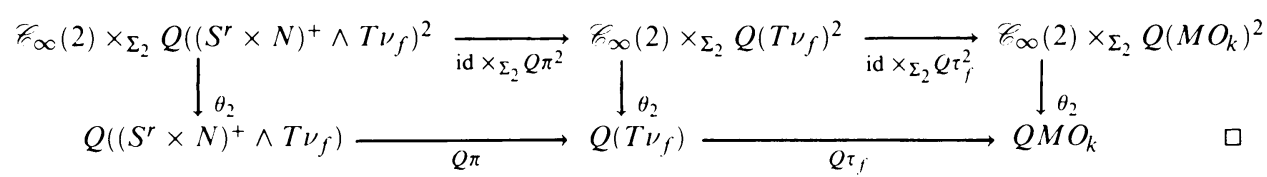

\subsection{Theorem. (a) The immersions}

$\widetilde{Q}_{i_{1}} \widetilde{Q}_{i_{2}} \cdots \widetilde{Q}_{i_{d}}\left[H\left(1, \alpha_{1}\right) \times H\left(1, \alpha_{2}\right) \times \cdots \times H\left(1, \alpha_{k}\right) \rightarrow \widetilde{D}\left(\mathscr{H}_{\alpha_{1}} \times \mathscr{H}_{\alpha_{2}} \times \cdots \times \mathscr{H}_{\alpha_{k}}\right)\right]$, for each sequence $0<i_{1} \leq i_{2} \leq \cdots \leq i_{d}, d \geq 0$, and each sequence $0 \leq \alpha_{1} \leq$ $\alpha_{2} \leq \cdots \leq \alpha_{k}$, are polynomial generators for $I(*, k), k>0$.

(b) The immersions

$$
\widetilde{Q}_{i_{1}} \widetilde{Q}_{i_{2}} \cdots \widetilde{Q}_{i_{d}}\left[P^{i_{1}} \times P^{\alpha_{2}} \times \cdots \times P^{\alpha_{k}} \rightarrow \tilde{D}\left(\gamma_{\gamma_{1}} \times \gamma_{r_{2}} \times \cdots \times \gamma_{\nu_{k}}\right)\right],
$$

for each sequence $0<i_{1} \leq i_{2} \leq \cdots \leq i_{d}, d \geq 0$, and each sequence $0 \leq \alpha_{1} \leq$ $\alpha_{2} \leq \cdots \leq \alpha_{k}$, are polynomial generators for $I(*, k), k>0$.

Proof. The theorem follows from 3.2, 2.12, 3.11 and 4.5.

\section{REFERENCES}

1. J. F. Adams, Infinite loop spaces, Ann. of Math. Studies, no. 90, Princeton Univ. Press, Princeton, N. J., 1978.

2. R. M. Alliston, Dyer-Lashof operations and bordism, Ph. D. Thesis, Univ. of Virginia, 1976.

3. T. Brocker and T. tom Dieck, Kobordisementheorie. Lecture Notes in Math., vol. 178, SpringerVerlag, Berlin and New York, 1970.

4. W. Browder, Homology operations and loop spaces, Illinois J. Math. 4 (1960), 347-357.

5. F. R. Cohen, J. P. May and T. Lada, The homology of iterated loop spaces, Lecture Notes in Math., vol. 533, Springer-Verlag, Berlin and New York, 1976.

6. P. E. Conner and E. E. Floyd, Differentiable periodics maps, Ergeb. Math. Grenzgeb. vol. 33, Springer-Verlag, Berlin and New York, 1964. 
7. __ Fibring within a cobordism class, Michigan Math. J. 12 (1965), 33-47.

8. E. Dyer and R. K. Lashof, Homology of iterated loop spaces, Amer. J. Math. 84 (1962), 35-88.

9. D. S. Kahn and S. B. Priddy, The transfer and stable homotopy theory, Math. Proc. Cambridge Philos. Soc. 83 (1978), 103-111.

10. J. P. May, The geometry of iterated loop spaces, Lecture Notes in Math., vol. 271, SpringerVerlag, Berlin and New York, 1972.

11. J. Milnor, On the Stiefel-Whitney number of complex manifolds and of spin manifolds, Topology 3 (1965), 223-230.

12. H. Schulte-Croonenberg, Dyer-Lashof Operationen in Bordismustheorien, $\mathrm{Ph}$. D. Thesis, Univ. of Wuppertal, 1981.

13. P. A. Schweitzer, Joint cobordism of immersions, Lecture Notes in Math., vol. 168, SpringerVerlag, Berlin and New York, 1970, pp. 267-282.

14. R. M. Switzer, Algebraic topology: homotopy and homology, Grundlehren Math. Wiss., Band 212, Springer-Verlag, Berlin, 1975.

15. R. Thom, Quelques propriétés globales des variétés différentiables, Comment. Math. Helv. 28 (1954), 17-86.

16. T. tom Dieck, Steenrod-Operationen in Kobordismehntheorien, Math. Z. 107 (1968), 380-401.

17. C. T. C. Wall, Cobordism of pairs, Comment. Math. Helv. 35 (1961), 136-145.

Instituto de Matemáticas, Universidad Nacional Autónoma de México, Ciudad Universitaria, México 04510, D. F., México 